\title{
High-Grade Cervical Dysplasia following Radiation Therapy for Invasive Cervical Cancer: A Report of Four Cases
}

\author{
Mila Pontremoli Salcedo ${ }^{a} \quad$ Andrea M. Milbourne ${ }^{b} \quad$ Anuja Jhingran ${ }^{c}$ \\ Patricia J. Eifel ${ }^{c}$ Pedro T. Ramirez ${ }^{b}$ Kathleen M. Schmeler ${ }^{b}$ \\ ${ }^{a}$ Department of Gynecology and Obstetrics, Federal University of Health \\ Sciences/Irmandade Santa Casa de Misericórdia de Porto Alegre, Brazil; Departments of \\ ${ }^{b}$ Gynecologic Oncology and Reproductive Medicine and ${ }^{\mathrm{C}}$ Radiation Oncology, The \\ University of Texas MD Anderson Cancer Center, Houston, Tex., USA
}

\section{Key Words}

Cervical dysplasia · Cervical cancer · Radiation therapy · Cold knife cone

\begin{abstract}
Introduction: The standard treatment for locally advanced cervical cancer is chemoradiation, with the majority of patients having a complete response to the therapy. The current surveillance recommendations from the Society of Gynecologic Oncology include annual cytology, with a small proportion of patients subsequently diagnosed with high-grade cervical dysplasia (CIN 2/3). To date, there is limited information regarding the optimal treatment and outcome for patients diagnosed with CIN 2/3. The current report describes the diagnosis, management and outcome of 4 patients diagnosed with CIN 2/3 following chemoradiation. Case Description: We describe 4 patients who developed CIN 2/3 seven months to 8 years following radiation therapy for locally advanced cervical cancer. All 4 patients were asymptomatic and the abnormalities were first detected by a Pap test. Three of the patients were managed conservatively with observation, and the CIN 2/3 resolved without intervention. One patient underwent 2 cervical conizations followed by a hysterectomy with no residual dysplasia noted on the hysterectomy specimen. Conclusion: The majority of patients with recurrent cervical cancer after chemoradiation are symptomatic, and most cases are detected by a physical examination. The role of cytology, colposcopy and biopsies may be of limited value. Furthermore, the significance of the diagnosis of CIN 2/3 in patients previously treated
\end{abstract}


Salcedo et al.: High-Grade Cervical Dysplasia following Radiation Therapy for Invasive Cervical Cancer: A Report of Four Cases

with radiation therapy was not associated with recurrent disease in the 4 patients described. Our results suggest that cytology may be of limited value in detecting recurrence in patients following radiation therapy, even when CIN 2/3 is detected.

(C) 2015 S. Karger AG, Basel

\section{Background}

The standard treatment for locally advanced cervical cancer is chemoradiation. It consists of external beam radiation therapy followed by brachytherapy, both given with concomitant weekly chemotherapy with cisplatin $[1,2]$. The majority of patients have a complete response to therapy and undergo surveillance. The current Society of Gynecologic Oncology (SGO) surveillance guidelines for patients following chemoradiation for locally advanced cervical cancer include pelvic examination and review of symptoms every 3 months for the first 2 years, every 6 months for years 3 through 5, and then annually. In addition, cytologic evaluation with a Pap test is recommended yearly for all patients [3].

Colposcopy was previously recommended for all patients with an abnormal Pap test following treatment for cervical cancer. However, the SGO has recently refined its recommendations through the 'choosing wisely' campaign and does currently not recommend performing colposcopy if the Pap test result is low-grade squamous intraepithelial lesion (LSIL) or less [4]. However, colposcopy and biopsies continue to be recommended for patients with a surveillance Pap test result of high-grade squamous intraepithelial lesion (HSIL). To date, there is limited information regarding the incidence, prognosis and recommended treatment for patients diagnosed with high-grade cervical dysplasia [cervical intraepithelial neoplasia (CIN) 2/3] following chemoradiation. In addition, it remains unclear if the evaluation and treatment of HSIL and CIN 2/3 in patients previously treated with chemoradiation is beneficial. The current report describes the diagnosis, management and outcome of 4 patients who developed CIN 2/3 seven months to 8 years following chemoradiation for locally advanced cervical cancer. Informed consent was obtained from the patients for the publication of this case series.

\section{Case Series}

Case 1

The patient is a 45-year-old woman with HIV who underwent chemoradiation for stage IIB moderately differentiated squamous cell carcinoma of the cervix. She had a complete response to therapy by physical examination and PET scan. Seven months following treatment, she developed vaginal bleeding. A pelvic examination showed an irregular-appearing cervix; an examination under anesthesia was performed, and multiple cervical biopsies were obtained. One biopsy showed CIN 2/3 with the remainder showing radiation-induced atypia. A cold knife cone (CKC) was then performed which showed CIN 2 with no invasive carcinoma identified. CIN 2 was present at the ectocervical margin, and the endocervical margin was uninvolved. It was recommended that the patient continue on surveillance. Repeat colposcopy and cervical biopsies 3 months later showed CIN 3. She underwent a second CKC that showed CIN 3 with a positive ectocervical margin and a negative endocervical margin. It was recommended that she undergo a third CKC, but it was not feasible due to minimal remaining cervix and radiation changes. She therefore underwent a robotic simple hysterectomy with bilateral salpingo-oophorectomy with no complications after the surgery. A final 
Salcedo et al.: High-Grade Cervical Dysplasia following Radiation Therapy for Invasive Cervical Cancer: A Report of Four Cases

pathology showed no residual dysplasia or malignancy. She is currently without any evidence of disease 6 months after surgery.

Case 2

The patient is a 35-year-old woman who underwent chemoradiation for stage IIB poorly differentiated squamous cell carcinoma of the cervix. She had a complete response and underwent surveillance with regular pelvic examinations and Pap tests. Eight years following treatment, a Pap test showed HSIL. Colposcopy was performed, and an acetowhite lesion was noted. A cervical biopsy revealed CIN 3. CKC was recommended, but the patient declined and pursued alternative therapies with green tea and curcumin vaginal suppositories. She has undergone repeat colposcopy and cervical biopsies on 2 occasions 3 months apart, showing only granulation tissue with no evidence of cervical dysplasia or invasive cancer.

Case 3

The patient is a 53-year-old patient treated with chemoradiation for stage IIB poorly differentiated adenocarcinoma of the cervix. A Pap test performed 3 months after completing treatment showed LSIL. Colposcopy and biopsies were performed that showed dysplastic squamous epithelium, favoring a high-grade lesion. The patient was felt to have radiationrelated changes and was observed. However, 22 months following the completion of chemoradiation, she had a Pap test result of HSIL. No cervical lesions were noted on colposcopy, and an endocervical curettage was performed showing CIN 2/3. The patient was again managed conservatively with observation. She has undergone regular repeat pelvic examinations with Pap tests and is without any evidence of dysplasia or recurrent cancer 42 months following treatment.

Case 4

The patient is a 27-year-old woman treated with chemoradiation for stage IB2 poorly differentiated squamous cell carcinoma of the cervix. A Pap test 2 years after completing treatment showed HSIL. Colposcopy and biopsies showed radiation changes without evidence of dysplasia, and she was observed. Five and a half years after completing treatment, a Pap test again showed HSIL. Colposcopy was performed, and the cervical os could not be well visualized due to radiation changes. Multiple cervical and vaginal biopsies were performed that showed CIN 3, and an invasive component could not be ruled out. A CKC was attempted, but the cervix was flush with the vagina and the endocervical canal could not be visualized. Multiple biopsies of the cervix and vagina were again performed and were negative. She has been followed with regular pelvic examinations and Pap tests and is without evidence of dysplasia or cancer 9 years following the completion of treatment.

\section{Discussion}

The main goal of surveillance following definitive therapy for cervical cancer is the early detection of isolated recurrences that can be treated through salvage surgery or radiation therapy. The majority of recurrences occur within the first $2-3$ years following treatment, and up to $75 \%$ are symptomatic. Although routine surveillance is recommended with pelvic examination and cytology, there are no prospective studies specifically addressing their efficacy in asymptomatic women. Bodurka-Bevers et al. [5] retrospectively evaluated 993 patients with stage IB cervical cancer undergoing surveillance, 532 treated with radiation ther- 
Salcedo et al:: High-Grade Cervical Dysplasia following Radiation Therapy for Invasive Cervical Cancer: A Report of Four Cases

apy and 461 with surgery. A total of 133 patients (13\%) developed recurrent disease. Of these, $114(86 \%)$ were symptomatic and $19(14 \%)$ were asymptomatic. All asymptomatic pelvic recurrences were diagnosed by pelvic examination. Cytology did not detect a single asymptomatic recurrence. The report did not address whether any of the patients were diagnosed or treated for high-grade dysplasia.

Rimel et al. [6] performed a retrospective multi-institution study that evaluated 4,167 cytology results from 929 women treated for cervical cancer. They found an abnormal cytology in 312 (34\%) women, representing 15\% (626/4,167) of all Pap tests. No recurrence or CIN 3 were detected by colposcopy in patients with cytology showing ASC-US or LSIL with no macroscopic lesion. As a result of an abnormal Pap test, 201 colposcopies in 135 women were performed. In 45 patients, CIN 2 or worse was diagnosed, including 25 patients with CIN 3 and 12 patients with invasive cancer. When treatment modalities were compared, it was noted that more patients had abnormal Pap tests after radiotherapy $(391 / 2,646,14.8 \%)$ compared to surgery $(133 / 1,521,8.7 \%)$.

A subsequent study by Zola et al. [7] evaluated 327 women with recurrent cervical cancer. Of the 164 asymptomatic women, recurrent disease was detected by cytology in only 5 patients (3\%). Elit et al. [8] performed a systematic review of 17 retrospective trials evaluating multiple surveillance methods in patients treated for cervical cancer. They found that 8$26 \%$ of patients developed recurrent disease. Asymptomatic recurrences were detected in $29-71 \%$ of patients using physical examination, but in only $0-17 \%$ of patients using cytology. The authors concluded that cytology does not add significantly to the detection of early disease recurrence in asymptomatic women. These low rates of detection have led to the recommendations by some authors to eliminate the use of cytology in surveillance or to limit its use to once a year $[3,4]$. Furthermore, the role of cytology in patients who have undergone pelvic radiation therapy may be even more limited and the elimination of its use from routine surveillance has been suggested.

There are limited data regarding the evaluation and management of patients undergoing surveillance who have abnormal cytology results. Current guidelines recommend against performing colposcopy in patients with a Pap test result of LSIL or less [4]. However, the management of patients with a Pap test result of HSIL and a subsequent diagnosis of highgrade dysplasia following radiation therapy has not been described. In this report, we describe 4 patients diagnosed with CIN 2/3 following chemoradiation. All 4 patients were asymptomatic, and the abnormalities were first detected by a Pap test. Three of the patients were managed conservatively with observation, and the CIN 2/3 resolved. One patient underwent two CKCs followed by hysterectomy with no residual dysplasia noted on the hysterectomy specimen. It does not appear that surveillance with cytology, even with a subsequent diagnosis of CIN 2/3, resulted in significant disease in these patients. Although no serious adverse events were noted, surgical intervention following radiation can be challenging and associated with serious complications including bleeding, infection, damage to surrounding organs, as well as rectovaginal and vesicovaginal fistulae.

\section{Conclusion}

The optimal surveillance methods for women following radiation therapy for locally advanced cervical cancer remains unclear. The majority of patients are symptomatic and most cases of recurrent disease are detected by physical examination. As evidenced by this case report, the role of cytology and subsequent colposcopy with cervical biopsies may be of limited value. Furthermore, the significance of the diagnosis of CIN 2/3 in patients previously 


\section{Case Reports in Oncology}

\begin{tabular}{l|l}
\hline \multicolumn{2}{l}{ Case Rep Oncol 2015;8:217-221 } \\
\hline DOI: 10.1159/000382117 & $\begin{array}{l}\text { ○ 2015 S. Karger AG, Basel } \\
\text { www.karger.com/cro }\end{array}$ \\
\hline
\end{tabular}

Salcedo et al.: High-Grade Cervical Dysplasia following Radiation Therapy for Invasive Cervical Cancer: A Report of Four Cases

treated with radiation therapy is unclear and was not associated with recurrent disease in the 4 patients described in this report. Our results suggest that cytology may be of limited value in detecting recurrence in patients following radiation therapy even when CIN 2/3 is detected. Further study is needed to determine the best predictors of recurrence in this patient population.

\section{Disclosure Statement}

The authors have no conflicts of interest to declare.

\section{References}

$\longrightarrow 1$ Morris M, Eifel PJ, Lu J, et al: Pelvic radiation with concurrent chemotherapy compared with pelvic and para-aortic radiation for high-risk cervical cancer. N Engl J Med 1999;340:1137-1143.

$\longrightarrow 2$ Chemoradiotherapy for Cervical Cancer Meta-analysis Collaboration (CCCMAC): Reducing uncertainties about the effects of chemoradiotherapy for cervical cancer: individual patient data meta-analysis. Cochrane Database Syst Rev 2010, CD008285.

-3 Salani R, Backes FJ, Fung MFK, et al: Posttreatment surveillance and diagnosis of recurrence in women with gynecologic malignancies: Society of Gynecologic Oncologists recommendations. Am J Obstet Gynecol 2011;204:466-478.

4 http://www.choosingwisely.org/doctor-patient-lists/society-of-gynecologic-oncology (accessed January 6, 2015).

5 Bodurka-Bevers D, Morris M, Eifel PJ, et al: Posttherapy surveillance of women with cervical cancer: an outcomes analysis. Gynecol Oncol 2000;78:187-193.

6 Rimel BJ, Ferda A, Erwin J, et al: Cervicovaginal cytology in the detection of recurrence after cervical cancer treatment. Obstet Gynecol 2011;118:548-553.

7 Zola P, Fuso L, Mazzola S, et al: Could follow-up different modalities play a role in asymptomatic cervical cancer relapses diagnosis? An Italian multicenter retrospective analysis. Gynecol Oncol 2007;107:S150S154.

8 Elit L, Fyles AW, Devries MC, et al: Follow-up for women after treatment for cervical cancer: a systematic review. Gynecol Oncol 2009;114:528. 